\title{
Catalyst Composition and Impurity-Dependent Kinetics of Nanowire Heteroepitaxy
}

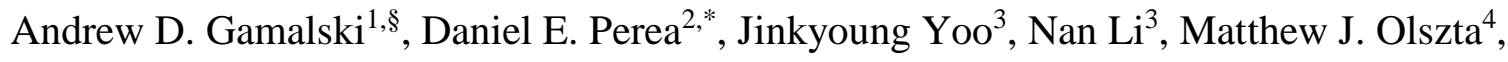 \\ Robert Colby ${ }^{2}$, Daniel K. Schreiber ${ }^{4}$, Caterina Ducati ${ }^{5}$, S. Tom Picraux ${ }^{3}$, Stephan Hofmann ${ }^{1, *}$ \\ ${ }^{1}$ Department of Engineering, University of Cambridge, Cambridge CB3 OFA, UK \\ ${ }^{2}$ Environmental Molecular Science Laboratory, Pacific Northwest National Laboratory, P.O. Box 999, Richland, Washington 99352, USA \\ ${ }^{3}$ Center for Integrated Nanotechnologies, Los Alamos National Laboratory, Los Alamos, New Mexico 87545, USA \\ ${ }^{4}$ Energy and Environment Directorate, Pacific Northwest National Laboratory, P.O. Box 999, Richland, Washington 99352, USA \\ ${ }^{5}$ Department of Materials Science and Metallurgy, University of Cambridge, Cambridge CB2 3QZ, UK
}

\begin{abstract}
The mechanisms and kinetics of axial Ge-Si nanowire heteroepitaxial growth based on the tailoring of the $\mathrm{Au}$ catalyst composition via $\mathrm{Ga}$ alloying are studied by environmental transmission electron microscopy combined with systematic ex-situ CVD calibrations. The morphology of the Ge-Si heterojunction, in particular the extent of a local, asymmetric increase in nanowire diameter, is found to depend on the Ga composition of the catalyst, on the TMGa precursor exposure temperature, and on the presence of dopants. To rationalize the findings, a general nucleation-based model for nanowire heteroepitaxy is established which is anticipated to be relevant to a wide range of material systems and device-enabling heterostructures.
\end{abstract}

Keywords: Nanowire, Ge-Si Heteroepitaxy, Nucleation Barrier, Environmental Transmission Electron Microscopy, AuGa Catalyst Alloy, Dopants

*Corresponding Authors: daniel.perea@pnnl.gov (D.E.P.), sh315@cam.ac.uk (S.H.)

${ }^{\S}$ Current Address: Intel Corporation, 5000 W. Chandler Blvd., Chandler, Arizona 85226, USA 
Heterojunctions play a central role in modern semiconductor devices, ranging from quantum well optoelectronics, ${ }^{1}$ two-dimensional electron gas devices ${ }^{2}$ and thermoelectric devices to tunnel field effect and high mobility, strained transistors. ${ }^{3}$ Small diameter semiconductor nanowires (NWs) offer an unprecedented range of new material combinations $^{4-6}$ to form device-relevant heterostructures, ${ }^{7-12}$ due to their ability to accommodate lattice-misfit strain ${ }^{13}$ and the ease of their integration via bottom-up growth. A key prerequisite is thereby the controlled formation of NW junctions during growth, in particular compositionally abrupt and structurally perfect axial NW heterostructures., ${ }^{9,14}$ Whereas sharp, defect-free heterojunctions can readily be achieved within group III-V NWs by vapor-liquid-solid (VLS) growth where the group V constituent typically has a low solubility in the conventionally used Au catalyst, ${ }^{15}$ this turns out to be much more challenging for group IV NWs with significantly higher solute solubilities. ${ }^{16}$ In particular for $\mathrm{Si} / \mathrm{Ge}$, the amount of growth solute in the Au catalyst nanoparticle, ${ }^{16}$ which acts as a reservoir during VLS growth, ${ }^{17}$ generally limits the junction abruptness, creating a current limitation to the further exploitation of the inherent advantages of these NWs. This reservoir effect can be minimized by catalyst alloying. ${ }^{5,18,19}$ A particular versatile process is thereby to alloy the catalyst via the vapor phase during chemical vapor deposition (CVD), e.g. the use of trimethylgallium (TMGa) to create AuGa alloy catalyst nanoparticles. ${ }^{19}$ Ga lowers the solubility of $\mathrm{Ge}$ and $\mathrm{Si}$ in the catalyst ${ }^{20}$ enabling sharper Ge-Si heterojunction NWs. However, currently there is limited understanding of the underlying mechanisms of such advanced axial heterostructure formation, the signature of which are unexpected side-effects such as the NW diameter becoming non-uniform at the Ge-Si heterointerface, creating a "bulge” (Fig. 1).

In this study we use environmental transmission electron microscopy (ETEM) to study in-situ the morphological details of Ge-Si NW heterojunction formation based on the tailoring of the Au catalyst composition via TMGa addition. We establish a nucleation-based model which allows us to rationalize the observed dependencies of bulging and related morphological changes at the Ge-Si interface with respect to the catalyst composition and the presence of impurities, such as dopants. Rather than just looking at the catalyst reservoir effect $^{17}$ and the surface energy balance at the heterointerface ${ }^{6}$ as in previous literature, we propose that the nucleation barrier for the heteroepitaxy, i.e. the Si precipitation on Ge, depends on the solubility of the growth species in the catalyst. The observed bulging is thereby a physical manifestation of the Si supersaturation in the alloy catalyst and reflects a transient change of surface energies. The presence of dopants can depress the nucleation 
barrier and consequently eliminate the formation of a bulge near the Ge-Si heterointerface. Hence the kinetics of heterointerface nucleation can be controlled through the catalyst composition and the addition of impurities during growth. Due to the generality of our arguments, we anticipate that our model is relevant to a wide range of material systems and device-enabling heterostructures, for some of which similar morphologies have been reported already. $^{21,22}$

\section{Results}

Figure 1 A-F shows post-growth scanning electron microscopy (SEM) and transmission electron microscopy (TEM) images of ex-situ grown Au-catalyzed Ge-Si heterojunctions, highlighting in particular the morphological differences (i.e. bulge size) based on the level of TMGa addition to the catalyst. In brief, the nanowire growth process consists of initial epitaxial GeNW growth using a $\mathrm{GeH}_{4}$ precursor, followed by the addition of TMGa to alloy the Au catalyst particle and then growth of a SiNW segment using $\mathrm{SiH}_{4}$ (see Methods). The addition of Ga into the liquid Au catalyst serves to decrease the Au-Ge eutectic composition (28 at.\% Ge for pure $\mathrm{Au}$; $~ 8$ at.\% for 2:1 Au:Ga alloy ${ }^{19}$ ), thus significantly lowers the concentration of Ge in the liquid catalyst prior to Si NW growth. This reduction of the reservoir of Ge atoms in the catalyst prior to Si growth leads to a much sharper Ge-Si compositional transition and details of this process have been demonstrated previously. ${ }^{19}$ Here we focus on the underlying mechanisms and kinetics of this heteroepitaxy, which for instance manifest themselves in the formation of a bulge at the Ge-Si interface, i.e. a locally, asymmetric increase in NW diameter at the heterointerface. Figure 1 shows that the relative size of this bulge increases with increasing Ga catalyst composition. Ge-Si NWs grown from elemental Au, without TMGa addition, show no bulge (Fig. 1B). Whereas a clear bulge is observed for $23 \%$ Ga (Figure 1C), which gets even more pronounced for $34 \% \mathrm{Ga}$ (Fig. 1D). ${ }^{24}$ These values refer to the Ga mole fraction in the catalyst alloy based on SEMand TEM-based EDX analysis of the catalyst particle. This bulge morphology is not an isolated curiosity, but rather is observed for $>90 \%$ of the NWs for a given sample. This reflects a conservative estimate of abundance, noting that for the remaining $10 \%$ of the NWs, a bulge may be present but is not visible due to the projected nature of the imaging. We note that smaller regularly spaced saw-tooth sidewalls can be a common feature of [111]-type wires, ${ }^{22}$ in particular for UHV based growth conditions, ${ }^{25}$ but the appearance of such a pronounced bulge has not been reported and more importantly its formation cannot be rationalized with previously reported growth models. 
The size of the bulge at the heterointerface also depends on the temperature at which the TMGa exposure and catalyst alloying is carried out. For a lower TMGa exposure temperature of $\sim 280^{\circ} \mathrm{C}$, the bulge becomes more pronounced and protrudes in a more radially-symmetric way (Fig. 1E). In addition, we find the size of the bulge to be also influenced by the presence of dopant gases during NW growth. Figure $1 \mathrm{~F}$ shows a Ge-Si heterojunction which was grown with an additional doping modulation from p-type Ge to ntype $\mathrm{Si}$, implemented using $\mathrm{B}_{2} \mathrm{H}_{6}$ and $\mathrm{PH}_{3}$ precursors during the growth of the respective $\mathrm{NW}$ segments (see Methods). No bulge is visible at the heterointerface for the many NWs observed, although the Ga fraction in the catalyst alloy was 20\%. A more in-depth discussion is given further below as to the influence of the dopants on the growth kinetics governing the formation of the observed bulge.

In order to gain unique insight into the formation of the observed bulge at the Ge-Si interface, we use an ETEM to observe in-situ the growth of the heterostructure from a Au-Ga alloy catalyst. Figures 2A-F shows a bright field ETEM image sequence of the catalytic GeSi NW heteroepitaxy. Pre-grown GeNWs with a Au catalyst particle at their tip were initially dispersed on a TEM grid and loaded in a modified Tecnai F20 ETEM operated at $200 \mathrm{kV}$ with a differential pumping system. The pre-grown NWs were exposed to TMGa in-situ to alloy the Au catalyst, followed by exposure to disilane to grow a SiNW segment. The catalyst composition is thereby estimated to be $\sim 34 \%$ Ga based on our ex-situ studies. ${ }^{24}$ The images in Figures 2A-F are representative of a video sequence (Video S1 in Supplementary Information) recorded at 9 frames per second. After exposure to TMGa, Figures 2A-B show a GeNW with a liquid AuGa catalyst alloy at the tip during disilane exposure, moments before the nucleation of a $\mathrm{Ge}_{1-x} \mathrm{Si}_{x}$ heterojunction. We note that the liquid AuGa catalyst/Ge interface (dashed line) is not planar, but truncated towards the triple phase boundary (TPB). We previously highlighted the importance of this TPB morphology in the context of the step flow cycle that constitutes axial NW growth. ${ }^{26}$ In particular the truncated edges act as a preferential attachment site for dissolved semiconductor material leading up to step nucleation, and thus provide a direct visualization of the level of supersaturation in the catalyst. $^{27-29}$ At $19 \mathrm{~s}$ a distortion is observed in the upper right of the projected TPB (Fig. 2C), leading to the filling of the truncated edge at the TPB (Fig. 2D), presumably with precipitated $\mathrm{Ge}_{1-x} \mathrm{Si}_{x}$. The limited time-resolution of the ETEM movie here does not allows us to resolve any oscillatory behavior of the truncated TPB morphology. However, we can clearly observe how after the initial precipitation event, NW growth accelerates and the Si segment 
temporarily increases in diameter (Fig. 2D/E) to then return to its original size as growth continues, leaving behind a small bulge (Fig. 2F). Note that we cannot directly measure the Ge composition after Ga exposure or the Si composition prior to the resumption of growth. However, as the NW did not appear to grow during TMGa exposure, we believe a significant fraction of the Ge remained in the catalyst prior to regrowth. In addition, since the NW diameters were all above $10 \mathrm{~nm}$, we do not expect a Gibbs-Thompson effect to significantly alter the alloy catalyst composition.

Despite different CVD conditions, the observation of a bulge from the in-situ data is consistent with ex-situ grown observations (Fig. 1, 2G). A fast Fourier transform (FFT) analysis of representative phase contrast TEM images (see inset Fig. 2G) shows that the upper and lower bulge facets are positioned approximately normal to the $[11 \overline{1}]$ and $[00 \overline{2}]$ directions. Hence we suggest that the bulge can be seen as an enlarged saw-tooth facet consisting of $\{111\} /\{100\}$ type planes on the [111]-orientated NWs. Supplemental Figure S1 gives further high resolution TEM analysis of the Ge-Si heterostructure (Fig. 2G) highlighting the as-grown crystalline quality.

Further to our ETEM data showing a truncated growth interface, post growth imaging of ex-situ grown NWs reveals a non-planar heterojunction interface for both intentionally doped and undoped NWs. Figure 3 compares ex-situ transmission electron microscopy data of an undoped Ge/Si heterojunction NW grown with a 34\% Ga alloyed catalyst (Fig. 3A) and a boron-doped Ge/phosphorus-doped Si heterojunction grown with a 20\% Ga alloyed catalyst (Fig. 3B). Details of the CVD growth process for these samples are described in Methods below. In both cases the Ge-Si interface shows a sharp compositional transition but is nonplanar with a clearly truncated edge. Whereas for the undoped NW a clear bulge can be seen in the Si region near the truncated edge (Fig. 3A), there is no such bulge for the doped NW (Fig. 3B). We note that for both the ex-situ doped and undoped NWs, only a single truncated edge is observed in projection. As shown in Fig. 2, the truncated edge forms at the TPB which serves as a preferential site for solute incorporation likely leading to an enhanced incorporation of dopants at the truncated edges as shown in a similar case by Connell et al. ${ }^{30}$ A formal discussion as to the formation of the truncated edge and the possible enhanced incorporation of dopants in this case is beyond the scope of this paper and will be addressed separately. 


\section{Discussion}

We next turn to a discussion of the kinetics governing the formation of the bulge for which we rely on three key observations from both the in-situ ETEM and ex-situ growth data. First, we observe that the size of the bulge is dependent on the Ga composition of the catalyst, the TMGa exposure temperature and the presence of dopants (Fig. 1G). Second, we find initially preferred $\mathrm{Ge}_{1-x} \mathrm{Si}_{x}$ precipitation at the truncated TPB edge. ${ }^{31}$ Third, the AuGa catalyst requires a long incubation time despite high precursor pressures. Our data, in particular the rapid NW growth that occurs after the nucleation of $\mathrm{Ge}_{1-x} \mathrm{Si}_{x}$ (i.e. after Figure 2B), does not support a possible reduced activity of the catalyst as the principle explanation for this long incubation time. ${ }^{19}$ We propose here the long incubation time indicates that a relatively high supersaturation in the AuGa catalyst is needed to nucleate $\mathrm{Ge}_{1-x} \mathrm{Si}_{x}$ on $\mathrm{Ge}$, suggesting the presence of a significant nucleation barrier for $\mathrm{Ge}_{1-x} \mathrm{Si}_{x}$ precipitation on the $\mathrm{Ge}$ NW. To reconcile all key observations, we introduce a model that couples the kinetics of GeSi heterostructure nucleation to catalyst composition and the presence of dopants during growth.

The premise of our model is centered on the nucleation barrier for the NW heteroepitaxy. As a consequence of the relatively low solubility of Ge in the AuGa alloy ${ }^{20}$ (the very aspect that produces a sharper Ge-Si transition relative to growth from elemental $\mathrm{Au}^{19}$ ), a more Si-rich $\mathrm{Ge}_{1-x} \mathrm{Si}_{x}$ layer must first nucleate at the liquid/solid interface of the Ge NW. We assert that the more Si-rich the layer to be nucleated, the higher is the expected nucleation barrier for heteroepitaxy. A widely used, simple model for heteroepitaxy of growing material $\mathrm{B}$ on $\mathrm{A}$ is to consider the balance of the relevant surface $\left(\sigma_{\mathrm{A}}, \sigma_{\mathrm{B}}\right)$ /interface $\left(\sigma_{i}\right)$ energies: $\Delta \sigma=\sigma_{B}+\sigma_{i}-\sigma_{A}$. A layer-by-layer growth mode is expected to occur for $\Delta \sigma<0$, whereas island growth of $\mathrm{B}$ on $\mathrm{A}$ is expected for $\Delta \sigma>0 .{ }^{32}$ This simple idea has been previously expanded to NW heteroepitaxy, ${ }^{6}$ noting that for any given material combination, layer-by-layer growth will lead to a straight NW morphology, while island growth will lead to a kinked morphology. We note that Si epitaxy on Ge is energetically unfavorable, ${ }^{33}$ i.e. $\Delta \sigma>0$. Nonetheless our data here shows straight SiNW segments to grow on GeNWs (Figs. 13). The existence of an energy barrier to deposit $\mathrm{Ge}_{1-x} \mathrm{Si}_{x}$ on $\mathrm{Ge}$, however, is consistent with our observation of a long catalyst incubation time, indicating that a high Si supersaturation is required to start growth. Hence an excess of Si must build up in the AuGa catalyst to nucleate $\mathrm{Ge}_{1-x} \mathrm{Si}_{x}$ layer-by-layer growth on the GeNW. 
We hypothesize that the solubility of Ge in the alloy catalyst is the key variable in the formation of the bulge and this is reinforced by two key experimental observations. First, the bulge size is proportional to the Ga composition in the catalyst where an increase in bulge size is observed with increasing Ga composition from $17 \%$ to $34 \%$ (Figure $1 \mathrm{G}$ ). Second, the TMGa exposure temperature (i.e. temperature at which the AuGa alloy catalyst is formed) influences the bulge size (Figure 1E), despite the catalyst still having $34 \%$ Ga. At $280{ }^{\circ} \mathrm{C}$ before TMGa exposure, we estimate the liquid AuGe catalyst to have $22.7 \%$ Ge (mole fraction), ${ }^{34,35}$ compared to $28.4 \%$ Ge at $380{ }^{\circ} \mathrm{C}$. Therefore, there is less Ge initially dissolved in the liquid AuGe catalyst at lower temperatures and adding Ga to the catalyst (via TMGa) results in an even lower Ge mole fraction in the catalyst nanoparticle. The low Ge composition of the catalyst following the TMGa exposure at $280{ }^{\circ} \mathrm{C}$ suggests an even larger barrier to nucleate $\mathrm{Ge}_{1-x} \mathrm{Si}_{x}$, requiring a larger Si supersaturation to restart NW growth, resulting in a larger bulge at the Ge-Si heterointerface (Figure $1 \mathrm{E}$ ) compared to $380{ }^{\circ} \mathrm{C}$. We thus infer that the bulge is a physical manifestation of the Si supersaturation in the liquid AuGa catalyst.

As highlighted in Figure 1F and 3B, with the addition of dopant gasses, it is possible to grow sharp Ge-Si heterojunctions without heterointerface deformities (i.e. without a bulge) from liquid AuGa catalyst particles. This suggests that the boron impurities do not raise the Ge solubility of the AuGa catalyst, and instead that the introduction of dopant impurities (phosphorous during Ge growth and boron during Si growth) lowers the energy penalty for $\mathrm{Ge}_{1-x} \mathrm{Si}_{x}$ precipitation on Ge, i.e. lowers the nucleation barrier for the heteroepitaxy. This hypothesis is supported by evidence which directly shows dopants to preferentially incorporate at the truncated edge of the TPB and suggests that impurities play a role in the nucleation process in VLS grown nanowires. ${ }^{36}$ Impurities are known to lower the nucleation barrier in phase transformations, depressing the barrier for homogeneous nucleation and reducing the supersaturation needed to achieve solidification. ${ }^{37}$ Such impurities are referred to as inoculants and are commonly used to speed up nucleation in undercooled melts. ${ }^{38}$ If impurities are lowering the nucleation barrier, then a lower supersaturation is required to nucleate $\mathrm{Ge}_{1-x} \mathrm{Si}_{x}$ resulting in a smaller bulge. Although the exact atomistic mechanisms by which dopants may lower the nucleation barrier remain unclear, we speculate the dopants may relax some of the strain in the $\mathrm{Ge}_{1-x} \mathrm{Si}_{x}$ cluster nuclei during precipitation on Ge. In order to reduce the formation of the bulge without the introduction of impurities, while at the same time be able to create a sharper junction, a suitable catalyst system is needed which lowers 
the solute solubility and simultaneously modestly changes the nucleation barrier. To our knowledge, no such catalyst system (pure or alloy) has been identified for VLS growth which will lead to abrupt junctions without the formation of a bulge. However, by switching to a VSS growth approach, this can be achieved as demonstrated by Wen et al. ${ }^{5}$

We note that transient changes in the surface/interface free energy terms could provide an alternative explanation for the observed bulge formation. In terms of Young's wetting equation ${ }^{37}$ a decrease in the liquid-vapor surface energy and/or increase in the liquidsolid surface energy during the Ge-Si transition would result in a decreased wetting angle, allowing the alloy droplet to cover a larger liquid-solid interface and to effectively increase the NW diameter (assuming the droplet's volume is constant). Our data shows, however, that the maximum of the bulge occurs at some point after the Ge-Si heterointerface when Si can be assumed to be the dominant dissolved semiconductor material in the catalyst. A simple surface energy argument thus fails to explain the observed heterointerface contour. Further, such argumentation is also inconsistent with the observed dependence on the TMGa exposure temperature (Fig. 1E). To explain our observations we propose that the initial high supersaturation needed to initiate heterostructure formation temporarily alters the surface energies, and consequently the equilibrium force balance at the TPB. Upon initiation of growth of the $\mathrm{Ge}_{1-x} \mathrm{Si}_{x}$ segment on $\mathrm{Ge}$, the high supersaturation in the liquid alloy droplet appears to either lower the liquid-vapor energy or raise the liquid-surface energy, decreasing the droplet wetting angle and causing the NW diameter to increase accordingly (see Fig. 2E). However, because the supersaturation in the droplet then decreases after nucleating $\mathrm{Ge}_{1-x} \mathrm{Si}_{x}$ on Ge, the surface energies return to their steady state growth values, resulting in the NW diameter to contract according to changed force balance at the TPB. The result of the initial heterostructure formation process is the formation of a "bulge" which has its maximum slightly after the actual heterointerface (see Fig. 2F).

In order to go beyond purely qualitative arguments, we employ a continuum nucleation model of depositing an $\mathrm{A}_{1-x} \mathrm{~B}_{x}$ alloy on pure $\mathrm{A}$ from a liquid catalyst $\mathrm{C}$ (analogous to depositing $\mathrm{Ge}_{1-x} \mathrm{Si}_{x}$ on $\mathrm{Ge}$ from a liquid AuGa catalyst). Note that we ignore the added complexities of step flow, 2D nucleation, and nanoscale effects. The surface energy difference $\Delta \gamma(x)$ of depositing $\mathrm{A}_{1-x} \mathrm{~B}_{x}$ on $\mathrm{A}$ is treated as a linear function of the material composition being deposited: $\Delta \gamma(x)=x \Delta \sigma$. Where $x=N^{\mathrm{B}} /\left(N^{\mathrm{A}}+N^{\mathrm{B}}\right)$ and $\Delta \sigma=\sigma_{\mathrm{B}}-\sigma_{\mathrm{A}}$. Note $\Delta \sigma>0$, as for Si growth on Ge. ${ }^{33} N^{\mathrm{A}}, N^{\mathrm{B}}$, and $N^{\mathrm{C}}$ are the number of A, B, and C atoms in the liquid catalyst respectively. Taking $\Delta \gamma(x)=x \Delta \sigma$ as the surface energy we can construct an 
expression for the Gibbs free energy change associated with nucleating $A_{1-\chi} B_{x}$ on pure $A$, Eqn. 1:

$$
\Delta G=\beta x \Delta \sigma(\Omega N)^{2 / 3}-\Delta \mu N
$$

Where $\Omega$ is the atomic volume of A or $\mathrm{B}, \beta$ is a geometric prefactor which governs the shape of the critical nucleus, and $N$ is the number of atoms in the $\mathrm{A}_{1-x} \mathrm{~B}_{x}$ cluster that will be precipitated on the A crystal surface. Treating the liquid as an ideal solution, the supersaturation is given by $\Delta \mu=k_{\mathrm{B}} T\left[(1-x) \ln \left(c^{\mathrm{A}}\right)+x \ln \left(c^{\mathrm{B}}\right)\right]-\mu_{\mathrm{s}}$. Where $c^{\mathrm{B}}=N^{\mathrm{B}} /\left(N^{\mathrm{A}}+N^{\mathrm{B}}+\right.$ $\left.N^{\mathrm{C}}\right)$ and $c^{\mathrm{A}}=N^{\mathrm{A}} /\left(N^{\mathrm{A}}+N^{\mathrm{B}}+N^{\mathrm{C}}\right)$ are the mole fraction of A and B in the liquid A-B-C catalyst respectively. $\mu_{\mathrm{S}}$ is the chemical potential of solid $\mathrm{A}_{1-x} \mathrm{~B}_{x}$. We rewrite Eqn. 1 to get the following dimensionless expression for the free energy, Eqn. 2:

$$
\frac{\Delta G}{k_{\mathrm{B}} T}=b x N^{2 / 3}-N\left[(1-x) \ln \left(c^{\mathrm{A}}\right)+x \ln \left(c^{\mathrm{B}}\right)-a\right]
$$

Where $a$ and $b$ are dimensionless coefficients defined as $a \equiv \mu_{\mathrm{S}} /\left(k_{\mathrm{B}} T\right)$ and $b \equiv$ $\left(\beta \Omega^{2 / 3} \Delta \sigma\right) /\left(k_{\mathrm{B}} T\right)$.

We want to understand how raising/lowering the composition of $\mathrm{A}$ in the catalyst, $c^{\mathrm{A}}$, and the dimensionless surface energy term $b$ determine if a nucleation barrier $\Delta G^{*} / k_{\mathrm{B}} T$ is present in Eqn. 2. The presence of a nucleation barrier is important, indicating a significant supersaturation must be reached to deposit $A_{1-x} B_{x}$ on $A$. Figure $4 A$ shows regions where a nucleation barrier is present for various values of $b$ and the cut off composition $c^{\mathrm{A}}$ where $\Delta G^{*} / k_{\mathrm{B}} T=0$. In the dark blue region of Figure $4 \mathrm{~A}$ there exists a barrier to nucleate $\mathrm{A}_{1-\chi} \mathrm{B}_{x}$ on A (i.e. $\Delta G^{*} / k_{\mathrm{B}} T>0$ ). In the white region of Figure $4 \mathrm{~A}$ no such barrier exists (i.e. $\Delta G^{*} / k_{\mathrm{B}} T=$ 0 ) and growth of $\mathrm{A}_{1-x} \mathrm{~B}_{x}$ on $\mathrm{A}$ proceeds readily, even under small supersaturations. The underlying free energy functions are plotted in Figure 4B-C for pathway $X-Y$ and $Y-Z$, respectively. Point $X$ in Figure 4A corresponds to an alloy catalyst rich in A and consequently no nucleation barrier. Traveling from $X$ to $Y$ illustrates how progressively higher supersaturations are needed to achieve nucleation and growth of $\mathrm{A}_{1-x} \mathrm{~B}_{x}$ on $\mathrm{A}$ as $c^{\mathrm{A}}$ falls. As $c^{\mathrm{A}}$ falls along pathway $X-Y$ we must nucleate more B-rich $\mathrm{A}_{1-x} \mathrm{~B}_{x}$ on $\mathrm{A}$ causing the energy barrier to rise as we approach point $Y$ (Figure 4A). Conversely, adding impurities (or other factors that alter the barrier for heteroepitaxy) corresponds to lowering the dimensionless surface term $b$ in our model system, i.e. pathway $Y$-Z (Figure 4A). Lowering $b$ has the opposite effect of reducing $c^{\mathrm{A}}$, namely lowering the nucleation barrier to deposit $\mathrm{A}_{1-x} \mathrm{~B}_{x}$ on $\mathrm{A}$. 
In Figure 5 we illustrate how the observed morphology of our Ge-Si heterostructured nanowires can be explained in the framework of our kinetic model (Eqn. 2 and Figure 4). In Figure 5 we again have points $X, Y$, and $Z$ which correspond to Ge-Si heterostructure formation under different growth conditions. Point $X$ corresponds to Ge-Si heterostructure growth from a Au catalyst (Figure 1B): as the Ge solubility in the catalyst is relatively high, Ge rich $\mathrm{Ge}_{1-x} \mathrm{Si}_{x}$ readily precipitates on the Ge NW with no significant nucleation barrier for heteroepitaxy and growth is possible at low supersaturations. Point $Y$ corresponds to lowering the Ge solubility in the catalyst through the creation of a AuGa alloy (Figure 1C-D). As the Ge catalyst composition falls we expect the nucleation barrier for $\mathrm{Ge}_{1-\chi} \mathrm{Si}_{x}$ precipitation to rise (as illustrated in our A-B model along pathway $X-Y$ in Figure 4A), requiring a higher supersaturation to achieve heteroepitaxial growth and producing a bulge in the NW. Point $Z$ corresponds to growing doped Ge-Si heterostructure NWs with AuGa catalyst particles (Figure 1F). In this case, the addition of the dopant impurities has reduced the overall energy penalty needed to achieve epitaxial growth, allowing sharp Ge-Si heterostructures without bulges at the heterointerface.

\section{Conclusions}

In conclusion, the mechanisms and kinetics of advanced axial Ge-Si nanowire heteroepitaxy based on the tailoring of the Au catalyst composition via TMGa exposure and the addition of dopants have been studied by environmental transmission electron microscopy combined with systematic ex-situ CVD experiments. The morphology of the Ge-Si heterojunction, in particular the extent of a local, pronounced bulge in the NW, is found to critically depend on CVD parameters, including the Ga composition of the catalyst, the TMGa exposure temperature, and the presence of dopants. Such pronounced NW bulging at a heterointerface has not been reported before, and more importantly it cannot be rationalized by prior simple models for heteroepitaxy, such as considering only the balance of the relevant surface/interface energies. For our Ge-Si model system, we infer that the NW bulging is a physical manifestation of the Si supersaturation in the liquid AuGa catalyst. We hypothesize that the lowered solubility of Ge in the AuGa alloy, i.e. the very aspect that produces an improved sharp Ge-Si transition, invokes a higher nucleation barrier for the heteroepitaxy based on the necessity of nucleating a more Si rich $\mathrm{Ge}_{1-x} \mathrm{Si}_{x}$ on the GeNW. The presence of dopants (specifically boron) can in turn depress this nucleation barrier, consequently enable the growth of sharp Ge-Si heterojunctions without heterointerface deformities. Our results 
highlight how the kinetics of NW heteroepitaxy can be controlled through the catalyst composition and the addition of impurities. While this work explores the control of the influence of nucleation kinetics over diameter and bulge formation with respect to the formation of heterojunctions, it is not possible to directly address the issue of heterojunction abruptness without additional studies. Due to the generality of our arguments, we anticipate that our nucleation based model for heteroepitaxy will be relevant to a wide range of material systems and device-enabling heterostructures.

\section{Methods}

In-situ TEM: A modified Tecnai F20 ETEM operated at $200 \mathrm{kV}$ with a differential pumping system and a digital video camera was used. Specimen heating was accomplished using a TEM holder mini furnace with a directly-attached thermocouple to measure the growth temperature. The ETEM experiments began with Au-catalyzed Ge nanowires grown ex-situ in a cold-walled chemical vapor deposition reactor at the Center for Integrated Nanotechnologies user facility at Los Alamos National Laboratory. The Ge NWs were subsequently transferred via sonication in isopropyl alcohol from the Si (111) substrate and dispersed onto a 200 mesh Cu TEM grid coated with both a holey carbon film and a $30 \mathrm{~nm}$ sputtered $\mathrm{SiO}_{x}$ layer. The samples were then exposed to a heat lamp for $30 \mathrm{~min}$ in air before loading into the ETEM for in-situ time-resolved imaging of Ge-Si heterojunction formation. To form the AuGa catalyst within the ETEM, the Au-catalyzed Ge NWs were heated to $380{ }^{\circ} \mathrm{C}$, and exposed to an atmosphere of $\approx 10$ mTorr of pure TMGa for $45 \mathrm{~s}$. Ga droplets were visible on the sputtered $\mathrm{SiO}_{x}$ (not shown) confirming the addition of Ga to the system. After forming the AuGa alloy, the Ge NWs with the AuGa catalyst were heated to $\approx 450{ }^{\circ} \mathrm{C}$ and exposed to $\approx 1$ Torr of pure $\mathrm{Si}_{2} \mathrm{H}_{6}$ to reinitiate growth.

Nanowire Growth (ex-situ $)^{19}$ : Both intrinsic Ge/Si axial heterostructured nanowires and boron-doped Ge/phosphorus-doped Si heterostructured nanowires were synthesized using $\mathrm{GeH}_{4}, \mathrm{SiH}_{4}, \mathrm{~B}_{2} \mathrm{H}_{6}$, and $\mathrm{PH}_{3}$ as the $\mathrm{Ge}, \mathrm{Si}, \mathrm{B}$ and $\mathrm{P}$ sources, respectively, and $\mathrm{H}_{2}$ as the carrier gas. Nanowire growth occurred on either $\mathrm{Si}(111)$ or $\mathrm{Ge}(111)$ substrates that were initially solvent-cleaned and native oxide etched prior to deposition of 30-50 nm Au colloid or e-beam deposited Au. Boron-doped $\mathrm{Ge}<111>$ nanowire growth $\left(\left[\mathrm{P}_{\mathrm{B} 2 \mathrm{H} 6}\right] /\left[\mathrm{P}_{\mathrm{Ge}}\right]=1.6 \mathrm{E}-4\right)$ was initiated 
from Au by nucleation at $380{ }^{\circ} \mathrm{C}$ for 3 minutes followed by growth at $280{ }^{\circ} \mathrm{C}$ for 90 minutes. In-situ catalyst alloying was accomplished during growth by ramping from $280{ }^{\circ} \mathrm{C}$ to $380{ }^{\circ} \mathrm{C}$ with $\mathrm{GeH}_{4}$ flow followed by simultaneously flowing vapor-phase trimethylgallium (TMGa) from a bubbler line and $\mathrm{GeH}_{4} ; \mathrm{B}_{2} \mathrm{H}_{6}$ was not flowing. The germanium nanowire growth at $380{ }^{\circ} \mathrm{C}$ results in unintentional Vapor-Solid (VS) sidewall growth leading to a marked increase in diameter and a tapered structure near the junction. The phosphorus-doped Si segment of the heterostructure was grown after $\mathrm{Au}_{1-\mathrm{x}} \mathrm{Ga}_{\mathrm{x}}$ alloy formation by removing both TMGa and $\mathrm{GeH}_{4}$ and introducing $\mathrm{SiH}_{4}$ and $\mathrm{PH}_{3}\left(\left[\mathrm{P}_{\mathrm{PH}}\right] /\left[\mathrm{P}_{\mathrm{Si}}\right]=6.6 \mathrm{E}-4\right)$, while the temperature was ramped from $380^{\circ} \mathrm{C}$ to $495^{\circ} \mathrm{C}$. A total pressure of 2 Torr was maintained throughout the Ge growth and alloy formation, while the total pressure of 0.5 Torr was maintained for the Si growth. Undoped Ge/Si heterostructures were also grown using the same procedure as for the doped wires with the exception that the Ge NWs were grown at $380{ }^{\circ} \mathrm{C}$. We note the possibility of Ga incorporation into the NW during growth analogous to what has recently been reported for pure $\mathrm{Al}$ catalysts, ${ }^{23}$ which could create a p-type region in the Si segment. Such possible minor Ga incorporation and related doping effects are not addressed here, but left as subjects for further studies. Ex-situ TEM imaging was performed on nanowires transferred to either copper grids with lacey carbon or to Si pillars using a dual-beam focused ion beam/scanning electron microscope.

\section{Acknowledgements}

S. H. acknowledges funding from ERC grant InsituNANO (no. 279342). A. D. G. acknowledges funding from the Marshall Aid Commemoration Commission and the National Science Foundation. C. D. acknowledges funding from the Royal Society. A portion of the research was also performed using EMSL, a national scientific user facility sponsored by the Department of Energy's (DOE) Office of Biological and Environmental Research and located at Pacific Northwest National Laboratory (PNNL). PNNL is operated by Battelle for the U.S. DOE under contract DE-AC05-76RL01830. We gratefully acknowledge the use of facilities within the LeRoy Eyring Center for Solid State Science at Arizona State University. This work was performed in part at CINT, a U.S. DOE, Office of Science User Facility. The research was funded in part by the Laboratory Directed Research and Development Program at LANL, an affirmative action equal opportunity employer operated by Los Alamos National 
Security, LLC, for the National Nuclear Security Administration of the U.S. DOE under contract DE-AC52-06NA25396.

\section{Supporting Information}

Bright field, 9 fps ETEM video showing Ge-Si NW heteroepitaxy (corresponding to Figs. 2A-F) and additional post growth TEM analysis of Ge-Si heterostructure shown in Fig. 2G. This material is available free of charge via the Internet at http://pubs.acs.org. 


\section{Figures}

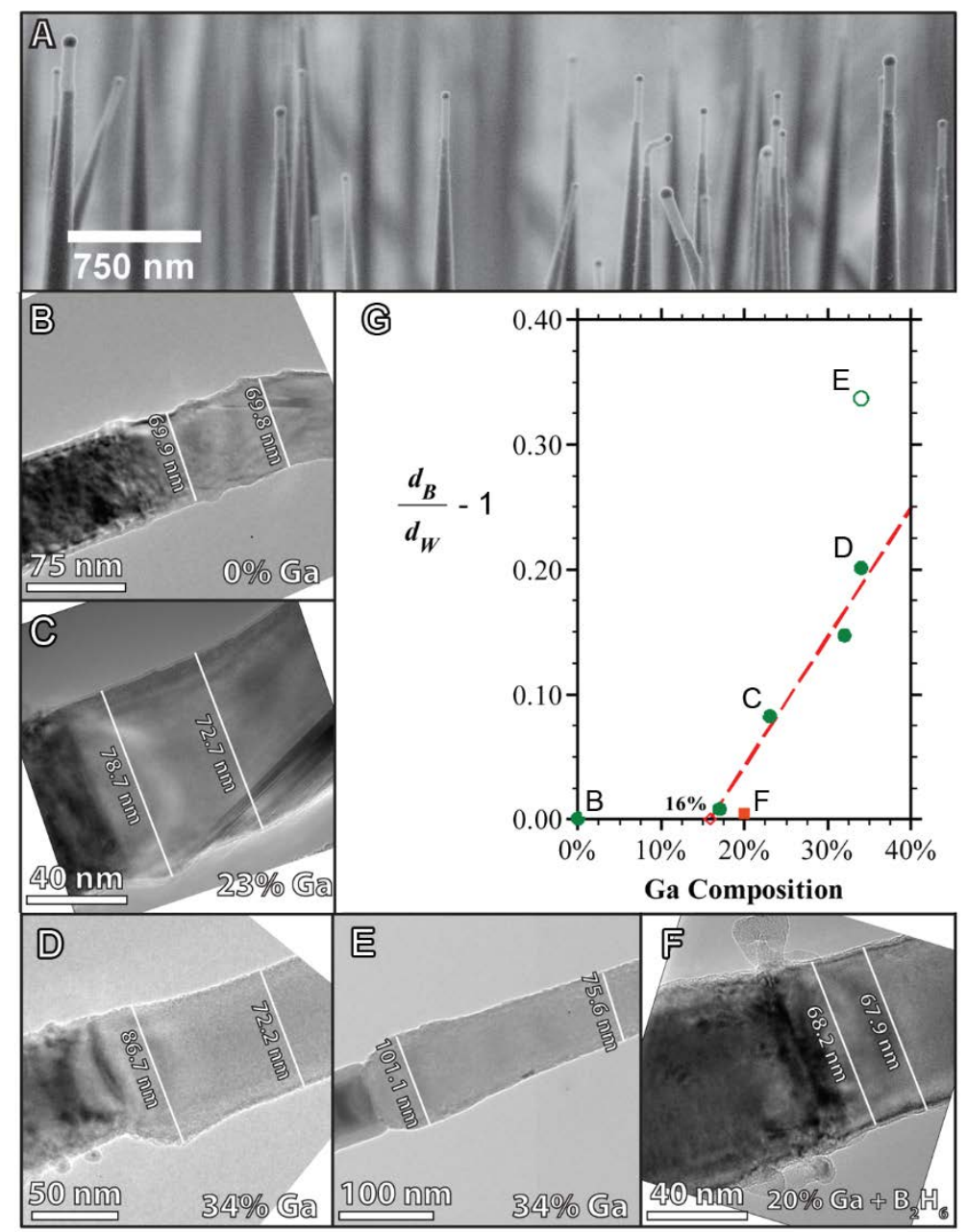

Figure 1: (A) A scanning electron microscope micrograph of undoped Ge-Si heterostructure NWs grown from $\mathrm{Au}_{0.68} \mathrm{Ga}_{0.32}$ catalysts. (B-D) Bright field post growth TEM micrographs of [111] oriented Ge-Si heterostructure NWs with 0\%, 23\%, and 34\% Ga (mole fraction) for B, $\mathrm{C}$, and $\mathrm{D}$ respectively. Ga composition was measured in the catalyst via EDX. TMGa exposure for B-D and F occurred at $380{ }^{\circ} \mathrm{C}$. (E) A Ge-Si heterostructure NW with a catalyst composition of $34 \%$ Ga (mole fraction) where TMGa exposure occurred at $280{ }^{\circ} \mathrm{C}$. (F) A n-p doped Ge-Si heterostructure NW with a catalyst containing 20\% Ga (mole fraction) based on EDX measurements from similar growth runs. The NW was doped in $\mathrm{B}_{2} \mathrm{H}_{6}$ for the Ge p-type segment and in $\mathrm{PH}_{3}$ for the Si n-type segment. (G) A plot of the increase (in dimensionless ratio) of the measured bulge to wire diameter plotted against Ga catalyst composition (mole fraction) in the catalyst. $d_{\mathrm{B}}$ is the diameter of the "bulge" and $d_{\mathrm{W}}$ is the diameter of the $\mathrm{Si}$ segment of the NW. For undoped Ge-Si heterojunction NWs grown under conditions of B-D (solid green circles), the $d_{\mathrm{B}} / d_{\mathrm{W}}-1$ vs. Ga composition plot fits well to a linear function at Ga catalyst compositions $>16 \%$. 




Figure 2: (A-F) Bright field ETEM image sequence representative of a video sequence (Video S1 in Supplementary Information) recorded at 9 frames per second in $\approx 1$ Torr of pure $\mathrm{Si}_{2} \mathrm{H}_{6}$ at $450{ }^{\circ} \mathrm{C}$. (G) Post growth bright field TEM image of a Ge-Si heterostructure grown ex-situ from a AuGa catalyst under similar conditions. The inset of G is the FFT of the Si segment of the NW (See Supplementary Figure S1 for further analysis). 



Figure 3: (A) A dark field STEM image of an undoped Ge-Si heterojunction NW with a $34 \%$ Ga catalyst. TMGa exposure temperature was $380^{\circ} \mathrm{C}$. Contrast in dark field STEM imaging is proportional to $\mathrm{Z}^{2}$, meaning Ge will appear bright and Si dark. (B) Bright field TEM image of doped Ge-Si heterojunction NW with a $20 \%$ Ga catalyst. While a truncated TPB region (arrow) is seen in both of these ex-situ growth cases, a bulge is only present in the undoped case. Note the spotty contrast in $\mathrm{B}$ is a result of unintentional $\mathrm{Pt} / \mathrm{C}$ deposition from specimen preparation using a dual-beam FIB/SEM. 



Figure 4: (A) A diagram showing how the composition of $\mathrm{A}$ in the catalyst and the value of $b$ determines if a non-zero nucleation barrier is present for heterostructure formation in our simplified $\mathrm{A}_{1-x} \mathrm{~B}_{x} / \mathrm{B}$ heteroepitaxy model. In these calculations $a=-3.0$ and $N^{\mathrm{C}}=1.0 \times 10^{6}$ atoms. The value for $a$ was calculated using the solid chemical potential for Ge at $500{ }^{\circ} \mathrm{C}$ from past literature ${ }^{34,}$. 35 (B,C) The free energy functions along path $X-Y$ and $Y-Z$, respectively. The colors of the free energy curves correspond to the points along the two paths given in (A). 




Figure 5: Schematic summary of the different observed heterointerfaces and growth scenarios. Pathways $X, Y$, and $Z$ schematically correspond to the points $X, Y$, and $Z$ in Figure 4. 
Table of Contents Figure

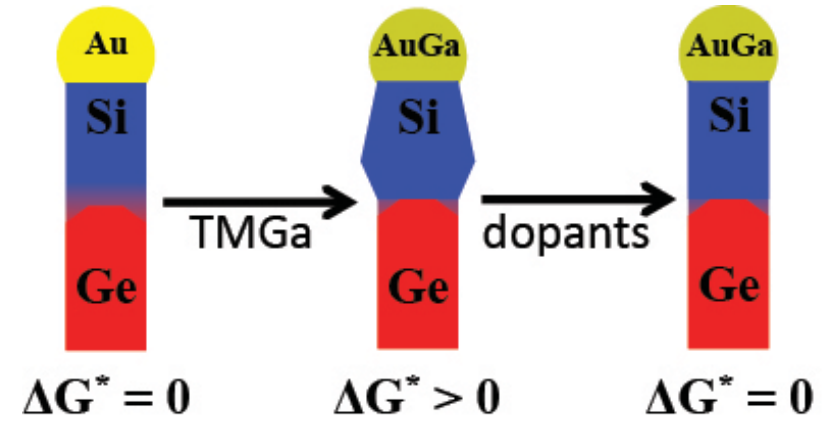




\section{References}

1. Nakamura, S.; Mukai, T.; Senoh, M., Candela-Class High-Brightness InGaN/AlGaN Double-Heterostructure Blue-Light-Emitting Diodes. Appl. Phys. Lett. 1994, 64, 16871689.

2. Adachi, S., GaAs, AlAs, and $\mathrm{Al}_{\mathrm{x}} \mathrm{Ga}_{1-\mathrm{x}} \mathrm{As}$ : Material Parameters for Use in Research and Device Applications. J. Appl. Phys. 1985, 58, R1-R29.

3. Fischetti, M. V.; Laux, S. E., Band Structure, Deformation Potentials, and Carrier Mobility in Strained Si, Ge, and SiGe Alloys. J. Appl. Phys. 1996, 80, 2234-2252.

4. Wu, Y.; Fan, R.; Yang, P., Block-by-Block Growth of Single-Crystalline Si/SiGe Superlattice Nanowires. Nano Lett. 2002, 2, 83-86.

5. Wen, C. Y.; Reuter, M. C.; Bruley, J.; Tersoff, J.; Kodambaka, S.; Stach, E. A.; Ross, F. M., Formation of Compositionally Abrupt Axial Heterojunctions in Silicon-Germanium Nanowires. Science 2009, 326, 1247-1250.

6. $\quad$ Dick, K. A.; Kodambaka, S.; Reuter, M. C.; Deppert, K.; Samuelson, L.; Seifert, W.; Wallenberg, L. R.; Ross, F. M., The Morphology of Axial and Branched Nanowire Heterostructures. Nano Lett. 2007, 7, 1817-1822.

7. Ionescu, A. M.; Riel, H., Tunnel Field-Effect Transistors as Energy-Efficient Electronic Switches. Nature 2011, 479, 329-337.

8. Björk, M. T.; Ohlsson, B. J.; Thelander, C.; Persson, A. I.; Deppert, K.; Wallenberg, L. R.; Samuelson, L., Nanowire Resonant Tunneling Diodes. Appl. Phys. Lett. 2002, 81, 4458-4460.

9. Gudiksen, M. S.; Lauhon, L. J.; Wang, J.; Smith, D. C.; Lieber, C. M., Growth of Nanowire Superlattice Structures for Nanoscale Photonics and Electronics. Nature 2002, 415, 617-620.

10. Borgström, M. T.; Verheijen, M. A.; Immink, G.; Smet, T. d.; Bakkers, E. P. A. M., Interface Study on Heterostructured GaP-GaAs Nanowires. Nanotech. 2006, 17, 40104013.

11. Jeppsson, M.; Dick, K. A.; Wagner, J. B.; Caroff, P.; Deppert, K.; Samuelson, L.; Wernersson, L.-E., GaAs/GaSb Nanowire Heterostructures Grown by MOVPE. J. Cryst. Grow. 2008, 310, 4115-4121.

12. Plissard, S. R.; Slapak, D. R.; Verheijen, M. A.; Hocevar, M.; Immink, G. W. G.; van Weperen, I.; Nadj-Perge, S.; Frolov, S. M.; Kouwenhoven, L. P.; Bakkers, E. P. A. M., From InSb Nanowires to Nanocubes: Looking for the Sweet Spot. Nano Lett. 2012, 12, 1794-1798.

13. Swadener, J. G.; Picraux, S. T., Strain Distributions and Electronic Property Modifications in Si/Ge Axial Nanowire Heterostructures. J. Appl. Phys. 2009, 105, 044310.

14. Li, D.; Wu, Y.; Fan, R.; Yang, P.; Majumdar, A., Thermal Conductivity of Si/SiGe Superlattice Nanowires. Appl. Phys. Lett. 2003, 83, 3186-3188.

15. Bjork, M. T.; Ohlsson, B. J.; Sass, T.; Persson, A. I.; Thelander, C.; Magnusson, M. H.; Deppert, K.; Wallenberg, L. R.; Samuelson, L., One-Dimensional Heterostructures in Semiconductor Nanowhiskers. Appl. Phys. Lett. 2002, 80, 1058-1060.

16. Clark, T. E.; Nimmatoori, P.; Lew, K.-K.; Pan, L.; Redwing, J. M.; Dickey, E. C., Diameter Dependent Growth Rate and Interfacial Abruptness in Vapor-Liquid-Solid $\mathrm{Si} / \mathrm{Si}_{1-\mathrm{x}} \mathrm{Ge}_{\mathrm{x}}$ Heterostructure Nanowires. Nano Lett. 2008, 8, 1246-1252.

17. Li, N.; Tan, T. Y.; Gösele, U., Transition Region Width of Nanowire Hetero- and pnJunctions Grown Using Vapor-Liquid-Solid processes. Appl. Phys. A 2008, 90, 591596. 
18. Chou, Y.-C.; Wen, C.-Y.; Reuter, M. C.; Su, D.; Stach, E. A.; Ross, F. M., Controlling the Growth of Si/Ge Nanowires and Heterojunctions Using Silver-Gold Alloy Catalysts. ACS Nano 2012, 6, 6407-6415.

19. Perea, D. E.; Li, N.; Dickerson, R. M.; Misra, A.; Picraux, S. T., Controlling Heterojunction Abruptness in VLS-Grown Semiconductor Nanowires via in situ Catalyst Alloying. Nano Lett. 2011, 11, 3117-3122.

20. Okamoto, H.; Prince, A.; Villars, P., Handbook of Ternary Alloy Phase Diagrams. ASM International: Materials Park, Ohio, 1995.

21. Hillerich, K.; Dick, K. A.; Wen, C. Y.; Reuter, M. C.; Kodambaka, S.; Ross, F. M., Strategies To Control Morphology in Hybrid Group III-V/Group IV Heterostructure Nanowires. Nano Lett. 2013, 13, 903-908.

22. Shin, N.; Chi, M.; Howe, J. Y.; Filler, M. A., Rational Defect Introduction in Silicon Nanowires. Nano Lett. 2013, 13, 1928-33.

23. Moutanabbir, O.; Isheim, D.; Blumtritt, H.; Senz, S.; Pippel, E.; Seidman, D. N., Colossal Injection of Catalyst Atoms into Silicon Nanowires. Nature 2013, 496, 78-82.

25. Ross, F. M.; Tersoff, J.; Reuter, M. C., Sawtooth Faceting in Silicon Nanowires. Phys. Rev. Lett. 2005, 95, 146104.

26. Hofmann, S.; Sharma, R.; Wirth, C. T.; Cervantes-Sodi, F.; Ducati, C.; Kasama, T.; Dunin-Borkowski, R. E.; Drucker, J.; Bennett, P.; Robertson, J., Ledge-FlowControlled Catalyst Interface Dynamics During Si Nanowire Growth. Nature Mater. 2008, 7, 372-375.

27. Oh, S. H.; Chisholm, M. F.; Kauffmann, Y.; Kaplan, W. D.; Luo, W.; Ruhle, M.; Scheu, C., Oscillatory Mass Transport in Vapor-Liquid-Solid Growth of Sapphire Nanowires. Science 2010, 330, 489-493.

28. Gamalski, A. D.; Ducati, C.; Hofmann, S., Cyclic Supersaturation and Triple Phase Boundary Dynamics in Germanium Nanowire Growth. J. Phys. Chem. C 2011, 115, 4413-4417.

29. Wen, C. Y.; Tersoff, J.; Hillerich, K.; Reuter, M. C.; Park, J. H.; Kodambaka, S.; Stach, E. A.; Ross, F. M., Periodically Changing Morphology of the Growth Interface in $\mathrm{Si}$, Ge, and GaP Nanowires. Phys. Rev. Lett. 2011, 107, 025503.

30. Connell, J. G.; Yoon, K.; Perea, D. E.; Schwalbach, E. J.; Voorhees, P. W.; Lauhon, L. J., Identification of an Intrinsic Source of Doping Inhomogeneity in Vapor-LiquidSolid-Grown Nanowires. Nano Lett. 2013, 13, 199-206.

32. Bauer, E., Phaenomenologische Theorie der Kristallabscheidung an Oberflaechen. Z . Kristallogr. 1958, 110, 372-394.

33. Marée, P. M. J.; Nakagawa, K.; Mulders, F. M.; Veen, J. F. v. d., Thin Epitaxial GeSi(111) Films: Study and Control of Morphology. Surf. Sci. 1987, 191, 305-328.

34. Chevalier, P. Y., A Thermodynamic Evaluation of the Au-Ge and Au-Si Systems. Thermochim. Acta. 1989, 141, 217-226.

35. Almazouzi, A.; Bernardini, J.; Moya, E. G.; Bracht, H.; Stolwijk, N. A.; Mehrer, H., Diffusion, Solubility and Thermodynamic Properties of Gold in Solid Germanium Studied by Means of Radiotracer and Spreading-resistance Analysis. J. Appl. Phys. 1991, 70, 1345-1354.

37. Porter, D. A.; Easterling, K. E.; Sherif, M. Y., Phase Transformations in Metals and Alloys. CRC Press: Boca Raton, FL, 2009.

38. McCartney, D. G., Grain Refining of Aluminium and Its Alloys Using Inoculants. International Materials Reviews 1989, 34, 247-260. 


\section{Notes}

24. The highest Ga composition we could achieve in the alloy catalyst was $34 \% \mathrm{Ga}$. Further exposure to TMGa does not lead to higher Ga compositions in the catalyst.

31. We do not speculate here about the detailed nucleation site(s) of step flow across the catalyst-NW interface, which remain experimentally undetermined.

36. Further discussion of the APT data in doped Ge-Si heterostructure NWs will be left for a future publication. 